\title{
A Study of Noncovalent Protein Complexes by Matrix-Assisted Laser Desorption/Ionization
}

\author{
Fenhong Song \\ Center for Advanced Research in Biotechnology, University of Maryland Biotechnology Institute, Rockville, \\ Maryland, USA
}

A sample preparation method has been developed for detection of noncovalent proteinprotein complexes by MALDI in this work. An aqueous solution of the matrix at $\mathrm{pH} 7$ allows the reproducible detection of a protein dimer, a protein tetramer, and a heterodimer. The signals are stable under long irradiation and can be detected at wide ranges of concentrations and with different laser intensities. (J Am Soc Mass Spectrom 2007, 18, 1286-1290) (c) 2007 American Society for Mass Spectrometry

$\mathrm{B}$ iomolecular interactions, such as protein-protein, protein-DNA, protein-RNA, and protein and small ligands, are involved in many biochemical processes. The development of biological mass spectroscopy, matrix-assisted laser desorption/ionization (MALDI) and electrospray ionization (ESI) provides us a new way to study these biomolecules and their interactions. ESI as a soft ionization technique has been used widely for the study of noncovalent interactions $[1,2]$. On the other hand, much less research has been done in this area by MALDI and it has generally been concluded that the overall approach is too harsh to allow detection of noncovalent protein complexes. The typical matrix for protein analysis is sinapinic acid in $30-50 \%$ of acetonitrile with $0.1 \%$ trifluoroacetic acid (TFA, $\mathrm{pH}<2$ ) and, in general, this condition would disrupt the noncovalent interaction of protein complexes during sample preparation. The laser desorption process itself may also dissociate some of the noncovalent complexes. Nevertheless, noncovalent complexes have been studied by MALDI with some success [3, 4].

The first demonstration of a noncovalent protein complex was reported by Hillenkamp's group, using nicotinic acid as the matrix dissolved in 10\% ethanol/ water [5]. The same group later reported the detection of protein complexes of the transmembrane Ompt porin, composed of three identical subunits, using ferulic acid dissolved in tetrahydrofuran (THF). They also reported the detection of protein complexes of streptavidin, alcohol dehydrogenase, and beef liver catalase using 2,6-dihydroxyacetophenone (DHAP) as the matrix in THF [6, 7]. Using different matrices and preparation techniques, studies of noncovalent complexes

Address reprint requests to Dr. Fenhong Song, U.S. Food and Drug Administration, Center for Food Safety and Applied Nutrition, 5100 Paint Branch Parkway, College Park, MD 20740, USA. E-mail: fenhong.song@fda.hhs.gov Current address: Center for Food Safety and Applied Nutrition, FDA, 5100 Paint Branch Parkway, College Park, MD 20740, USA. between peptides and DNA, RNA have been demonstrated [8-10]. Juhasz and Biemann [11] detected nonspecific noncovalent complexes of oligosaccharides with some basic peptides. Standard matrices buffered to physiological $\mathrm{pH}$ were found to be helpful to retain the intact protein structure. Cotter et al. [12] detected an enzyme-peptide complex using a saturated solution of sinapinic acid in 1/1 ethanol-1 $\mathrm{M}$ ammonium citrate. The human immune farnesyl protein transferase has been detected as a heterodimer by MALDI using sinapinic acid in acetonitrile-0.2 M Bis-tris (30:70) $(\mathrm{pH} 7)$ [4]. The complex between $\beta$-lactoglobulin and polyclonal anti- $\beta$-lactoglobulin antibody was detected by MALDI using sinapinic acid as the matrix when it was dissolved in $\mathrm{CH}_{3} \mathrm{CN} / \mathrm{H}_{2} \mathrm{O}(1: 1, \mathrm{vol} / \mathrm{vol})$ without addition of TFA [13]. When 6-aza-2-thiothymine (ATT) was used without addition of any organic solvent, the intact noncovalent protein complex, RNAse $\mathrm{S}$, as well as the noncovalent complex between S-protein, S-peptide, and specific dimers of coiled-coil leucine zipper polypeptide were observed [14].

Recent work showed that intact hemoglobin complex from whole human blood was detected by MALDI using a saturated solution of 2,6-DHAP in acetonitrile and $20 \mathrm{mM}$ ammonium acetate $1 / 3$ (vol/vol) [15]. However, no general methods for the observation of noncovalent complexes have been found and the applicability of different matrices to any given complexes in the literature reports is often inconsistent. In this work, a new sample preparation method at physiological $\mathrm{pH}$ was reported. The method uniquely uses sample preparation in aqueous solution at $\mathrm{pH}$ 7. Although the aqueous solution of ATT has been used at a $\mathrm{pH}$ close to 7 [14], this DNA matrix is not very effective for protein and protein complexes analysis. The method described here could be broadly applicable for the detection of protein-protein complexes. A preliminary study of oli- 
gomeric states of protein complexes prepared using this method is presented in this work.

\section{Experimental}

The experiments were performed using Voyager-DE (Applied Biosystems, Framingham, MA) MALDI-TOF instrument equipped with a 337-nm nitrogen laser. The flight path length of the instrument is $1.2 \mathrm{~m}$. All mass spectra were acquired in positive-ion linear mode with an acceleration voltage of $25 \mathrm{kV}$. The delay time was varied in the ranges from 450 to 1000 ns. Mass spectra were obtained by averaging 50 laser shots. Sinapinic acid, ammonium citrate, and avidin were purchased from Aldrich (Milwaukee, WI) and used without further purification. The matrix solution was prepared by adding $25 \mathrm{mg}$ of sinapinic acid and $50 \mathrm{mg}$ ammonium citrate in $0.5 \mathrm{~mL}$ of deionized water to give a saturated solution, then adjusted to $\mathrm{pH} 7$ with about $7 \mu \mathrm{L}$ of concentrated ammonium hydroxide. Avidin was dissolved in deionized water to yield a protein concentration of $80 \mu \mathrm{M}$. This solution was diluted with equal volume of matrix solution to give the final concentration $(40 \mu \mathrm{M})$. The class II major histocompatibility complex (MHC) is a gift from Dr. Roy Mariuzza's group. It was supplied as a aqueous solution containing $40 \mu \mathrm{M}$ protein, $20 \mathrm{mM}$ Tris- $\mathrm{HCl}$, and $20 \mathrm{mM}$ sodium chloride. It was diluted two or four times with matrix solution for a typical experiment. Pseudomonas aeruginosa PhzD was obtained courtesy of Dr. James Parsons, supplied as a solution with a protein concentration of 10 $\mathrm{mg} / \mathrm{mL}$ in $50 \mathrm{mM}$ Tris- $\mathrm{HCl}, 0.1 \mathrm{M}$ of $\mathrm{NaCl}, 1 \mathrm{mM}$ DTT, and $1 \mathrm{mM}$ EDTA. It was diluted with matrix solution to a concentration of $20 \mu \mathrm{M}$ for a typical experiment. The instrument was calibrated using horse myoglobin. Typically, four sample spots were prepared by loading $1 \mu \mathrm{L}$ of the analyte mixture. Four sample spots were deposited on a 100-well plate and multiple measurements were taken to verify the reproducibility of the results.

\section{Results and Discussion}

Noncovalent complexes of biological importance are normally formed at physiological or near neutral $\mathrm{pH}$. Although formation of noncovalent complexes in the presence of a neutral $\mathrm{pH}$ and aqueous solvent may not be the only factors for desorption of intact protein complexes in MALDI, in general it should be one of the prerequisites for the process. Thus acidic MALDI matrices and the presence of organic solvent may play a role in the dissociation of these complexes and prevent their observation. In this work, aqueous solutions of acidic matrices including 2,5-dihydroxybenzoic acid, $\alpha$-cyano-4-hydroxycinnamic acid, and sinapinic acid were neutralized to raise the $\mathrm{pH}$ to a range that does not interfere with noncovalent complexes formation.

Two approaches were tried in this work. First the matrices were simply adjusted to $\mathrm{pH} 7$, using a volatile base such as ammonium hydroxide. These were then mixed with samples and MALDI spectra were obtained. This yielded no spectrum of complexes. In the second approach, a volatile buffer such as ammonium citrate was added to the matrix, and the matrix/buffer mixture was then adjusted to $\mathrm{pH} 7$ with ammonium hydroxide. No organic solvents were added as cosolvents in these experiments. The later approach, which uses aqueous solution at physiological $\mathrm{pH}$, more specifically aqueous solution of sinapinic acid with ammonium citrate adjusted to $\mathrm{pH} 7$ with ammonium hydroxide, is very effective for the study of noncovalent protein-protein interactions by MALDI as subsequently shown. This sample preparation method gives very homogeneous analyte distribution, and the simple "dried-droplet" was used and found to be sufficient to produce spectra of complexes [3]. The results for three protein complexes are summarized here.

Pseudomonas aeruginosa PhzD is an isochorismatase from the phenazine biosynthetic pathway. The crystal structure has been solved and it is a dimer in the solid state. The solution molecular mass of this enzyme was determined by a combination of light scattering and interferometric refractometry [16] and it is also a dimer in solution. The MALDI spectrum of this enzyme (Figure 1), using sample preparation described here, showed a major peak of homodimer at $\mathrm{m} / \mathrm{z} 50 \mathrm{~K}$ and a major peak at $25 \mathrm{~K}$ of monomer with minor peaks from other high-order oligomers such as trimer and tetramer.

The second sample tested is avidin. Avidin is a glycoprotein found in egg white whose active form is a tetramer composed of identical subunits. Each subunit contains 128 amino acids [17]. The spectrum (Figure 2) showed the tetramer peaks at $m / z 63.1 \mathrm{~K}$ as the major peak in addition to the monomer at $m / z 15.7 \mathrm{~K}$ and a minor dimer peak at $\mathrm{m} / \mathrm{z} 31.7 \mathrm{~K}$. A trace amount of trimer peak was also observed. These lower-order oligomers are probably from the dissociation of the tetramer under the laser irradiation. However, the fact that the tetramer is the predominant species suggests that the basic solution structure of avidin is retained. These results are consistent with previous electrospray [18] and MALDI work [19] where it was also observed as a tetramer.

The third sample tested (Figure 3) is a class II major histocompatibility complex (MHC), a known heterodimeric protein with noncovalent $\alpha$ chain $(\mathrm{m} / z 22 \mathrm{~K})$ and $\beta$ chain $(m / z 21 \mathrm{~K})$ bound with a peptide $(\mathrm{m} / \mathrm{z} 1.5 \mathrm{~K})$ [20]. MHC proteins are cell surface proteins that serve as restricting elements for the cell-meditated immune system. The peak from the expected heterodimer was observed at $m / z 43 \mathrm{~K}$. The peptide is not observed as part of the complexes and may have been dissociated from the complex in the process of desorption and ionization.

To verify that the spectra of multimeric complexes observed above bear some relationship to their structure at physiological $\mathrm{pH}$ and are sensitive to the MALDI preparation conditions, the spectra of the three samples using a more conventional condition (sinapinic acid in $30-50 \%$ of acetonitrile with $0.1 \%$ of TFA) were also 


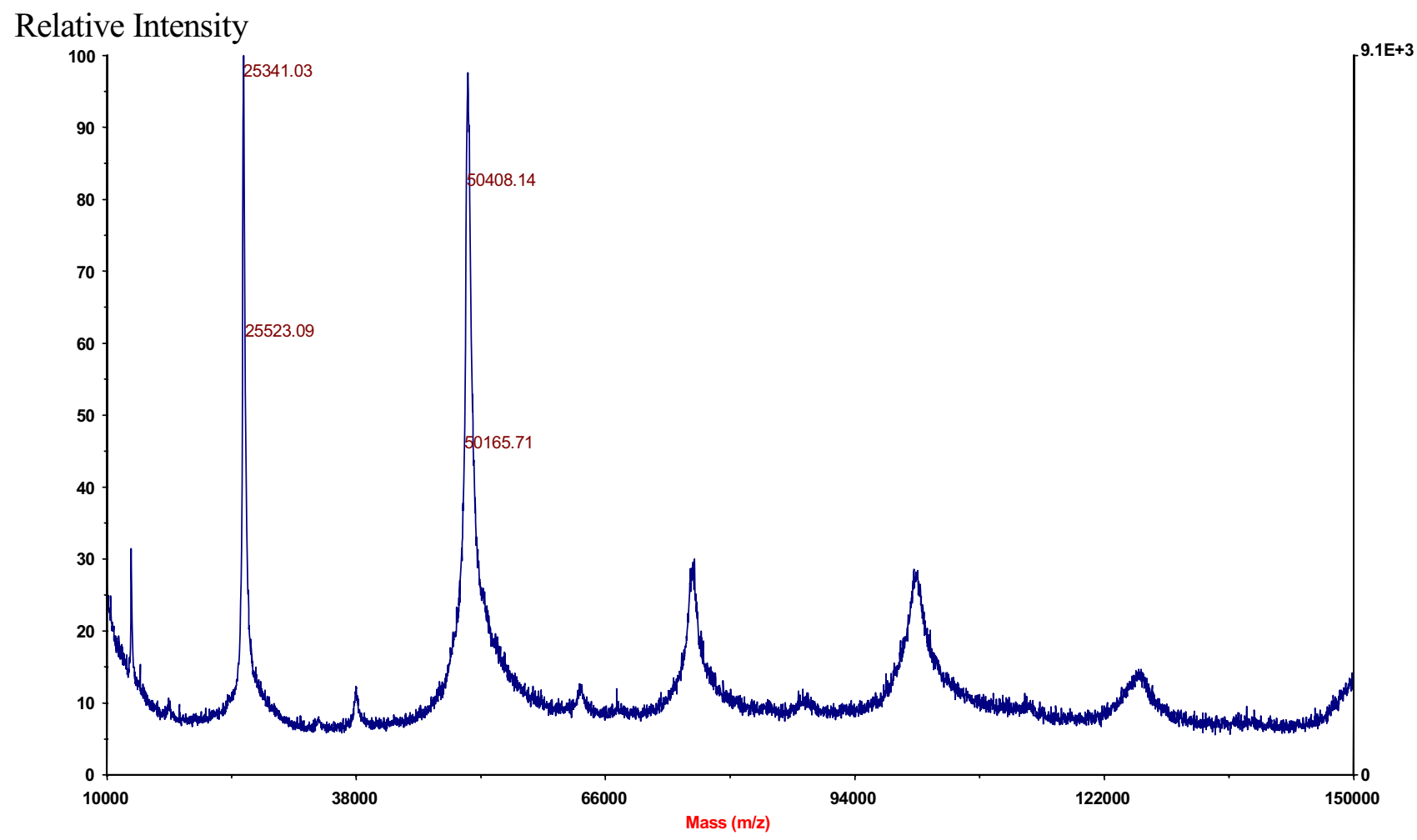

Figure 1. MALDI spectrum of PhzD $(20 \mu \mathrm{M})$.

acquired. In each case, the mass spectra showed monomer as the major peak and only minor high-order oligomeric peaks with decreasing intensity. Strong peaks corresponding to specific complex associations were not observed by MALDI under standard conditions, in contrast to the case where samples were

\section{Relative intensity}

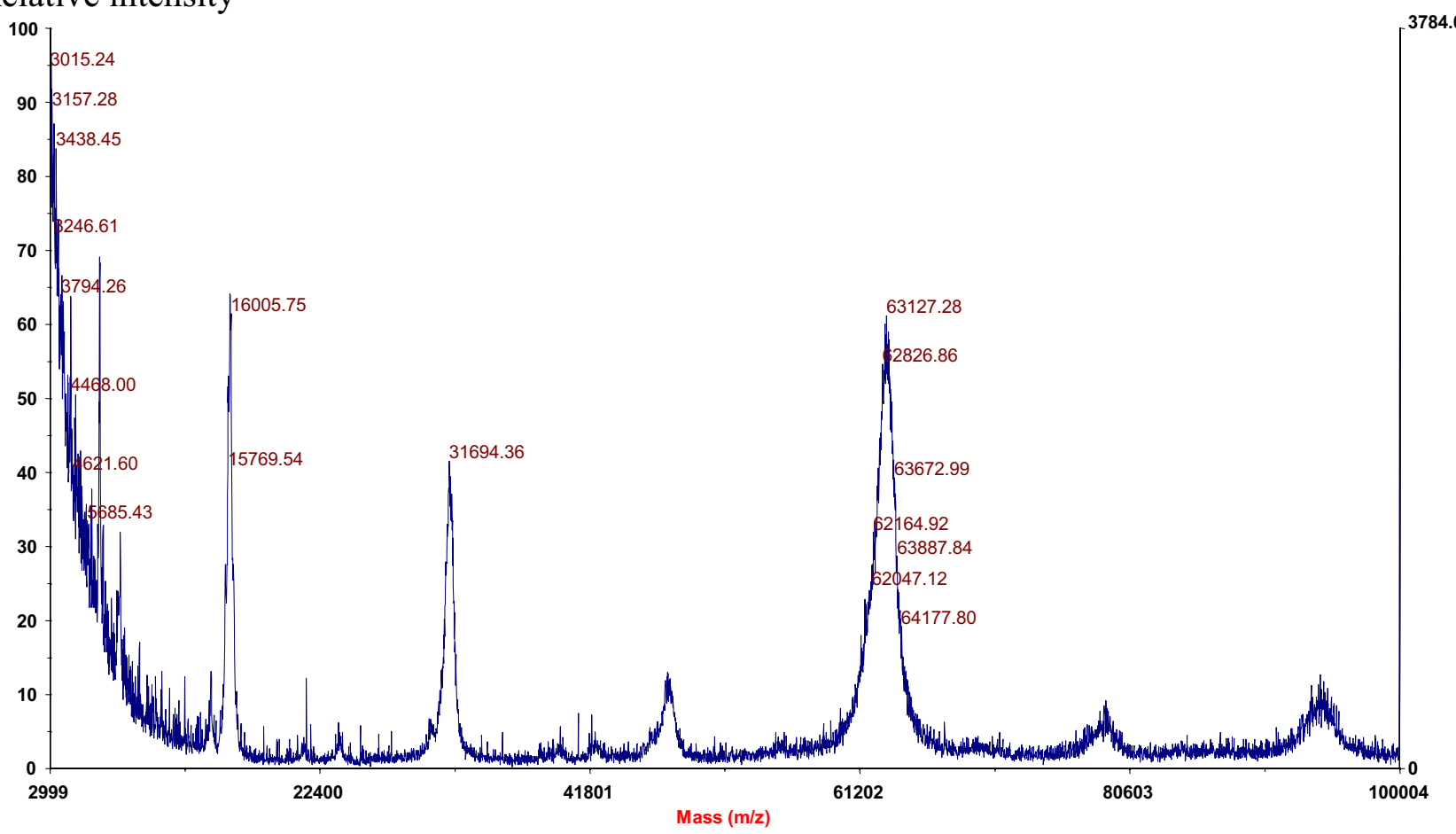

Figure 2. MALDI spectrum of avidin $(40 \mu \mathrm{M})$. 


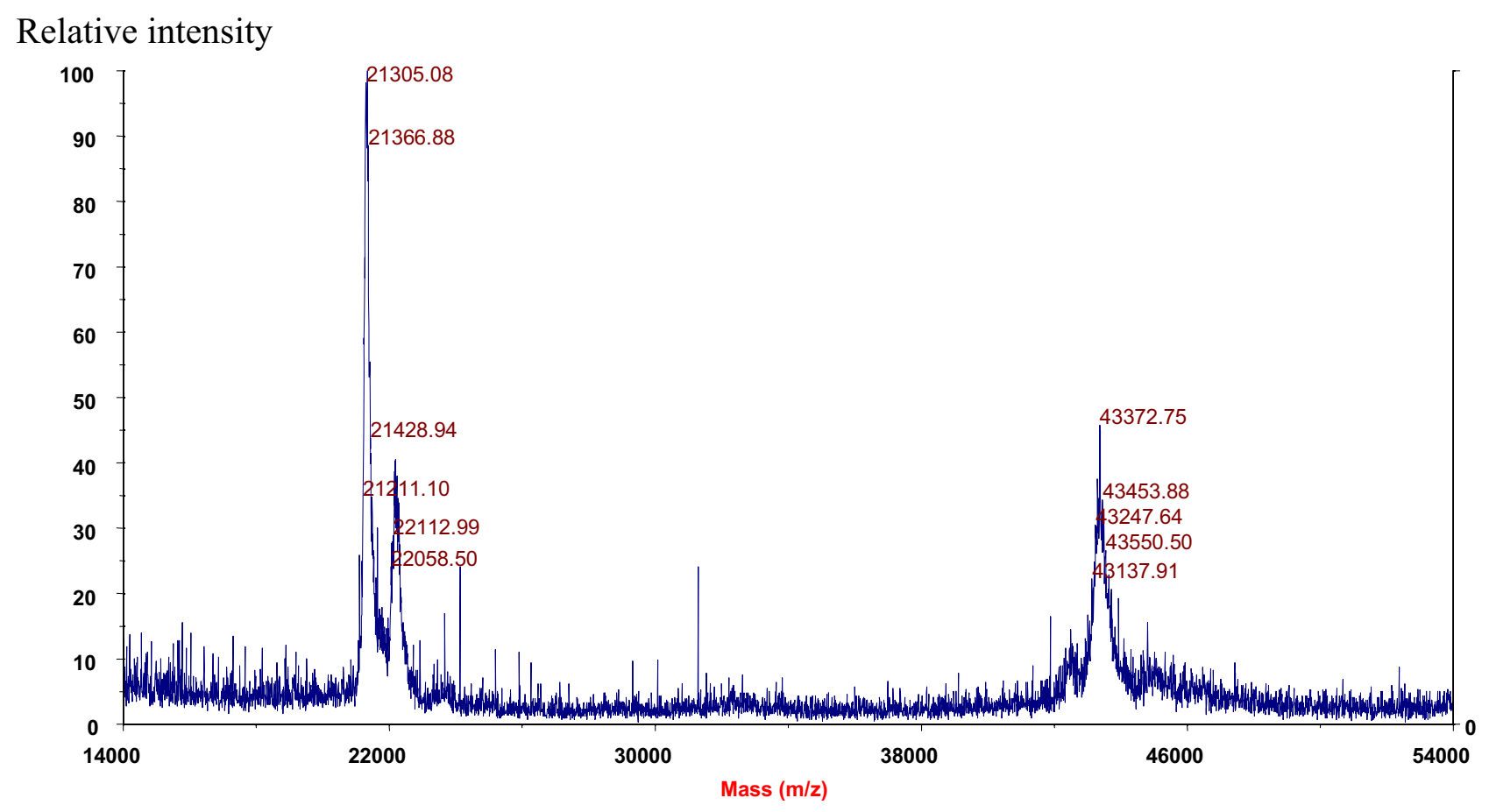

Figure 3. MALDI spectrum of a class II major histocompatibility complex (20 $\mu \mathrm{M})$.

prepared at neutral $\mathrm{pH}$. Nonspecific aggregates are often observed under MALDI conditions [4], but the signal intensity of nonspecific oligomers decreases approximately exponentially with increasing monomer number of the aggregates. The results of this study show that there is a distinct difference in the spectra observed using the standard methods where decreasing oligomer intensity is observed with increasing oligomer size and the method described here, in which the intensity of specific oligomers (e.g., the avidin tetramer) strongly suggests a relationship to biological structure.

The sample preparation method used here retains biological relevant intermolecular interaction and thus enhances the robustness of MALDI method for observation of these complexes. For example, the ionization of PhzD was also investigated under different laser intensities and at different concentrations. Higher laser intensities ( $50 \%$ higher than threshold) did not destroy the signals from the noncovalent complexes. The significant dimer peaks of PhzD can also be detected over a wide concentration range from 1 to $50 \mu \mathrm{M}$, although a concentration of $20 \mu \mathrm{M}$ was used for this protein in a typical experiment. The signals from noncovalent interaction in this work can be detected with repeated laser shots. The effect of repeated irradiation on the signals has been examined by few groups. Hillenkamp and coworkers [7] observed the so-called first shot phenomenon, in which prominent signals for the intact protein can be obtained from only the first laser shot on a not-yet-irradiated sample spot. Repeat irradiation caused the dissociation of complex in their studies. On the other hand, there are also other reports that stable signals were observed after long irradiation [21-23].
The success of this method for observation of noncovalent complexes, relative to most other methods for analysis of proteins by MALDI, may be because it does not rely on high concentrations of organic solvent such as acetonitrile and methanol for crystal formation. It also does not rely on incorporation of the analyte with low $\mathrm{pH}(0.1 \%$ of TFA or similar acids). For most protein complexes, these conditions would disrupt the noncovalent interactions. This is probably explains why protein complexes are not routinely observed by MALDI in the past, although some successes have been reported.

A major advantage of using this method is that it can be easily used to study complexes formed from two or more different proteins [24]. Thus, this approach now may offer a routine alternative to and significant advantage over electrospray approaches. The spectra of protein-protein interactions of three or more components by ESI-MS are complicated with multiply peaks from each unit [25]. With singly charged peaks, MALDI spectra are easily interpreted. Extensive desalting and exchanging buffer are often needed to study protein complexes by ESI-MS, unlike the MALDI approach. Finally, MALDI offers a significant advantage in speed, sensitivity, and accuracy compared with other techniques to study protein-protein interaction such as NMR, X-ray crystallography, size-exclusion chromatograph, sedimentation equilibrium ultracentrifugation, and nondenaturing gel electrophoresis.

\section{Conclusions}

Several different noncovalent protein-protein complexes have been consistently detected by MALDI using 
a sample preparation method reported in this work. This method uses aqueous solution at physiological $\mathrm{pH}$, which can be broadly used to retain physiologically relevant protein-protein complexes during sample preparation. The failure to observe these same complexes with conventional sample preparation methods (using organic solvent and at low $\mathrm{pH}$ ) further suggests that this sample preparation method retains the structure of physiologically relevant complexes. In general, good correlation was observed between the gas-phase protein complexes detected by MALDI and their known associations by other methods. High laser energy did not dissociate the protein complexes. The results indicate that the protein complexes can be relatively stable in gas phase and retained at least some of the quaternary structure. The results also suggest that MALDI could be generally used to study protein quaternary structures using the method reported here.

\section{Acknowledgments}

The author thanks Dr. John Callahan at FDA for his help with the writing, Dr. Edward Eisenstein at the University of Maryland for his support for this work, and University of Maryland Biotechnology Institute for financial support.

\section{References}

1. Loo, J. A. Studying Noncovalent Protein Complexes by Electrospray Ionization Mass Spectrometry. Mass Spectrom. Rev. 1997, 16, 1-23.

2. Heck, A. J. R.; van del Heuvel, R. H. H. Investigation of Intact Protein Complexes by Mass Spectrometry. Mass Spectrom. Rev. 2004, 23, $368-$ 389.

3. Bolbach, G. Matrix-Assisted Laser Desorption/Ionization Analysis of Non-covalent Complexes: Fundamentals and Applications. Curr. Pharm. Des. 2005, 11, 2535-2557.

4. Farmer, T. B.; Caprioli, R. M. Determination of Protein-Protein Interactions by Matrix-Assisted Laser Desorption/Ionization Mass Spectrometry. J. Mass Spectrom. 1998, 33, 697-704.

5. Karas, M.; Bahr, U.; Ingendoh, A.; Nordhoff, E.; Stahl, B.; Strupat, K.; Hillenkamp, H. Principles and Applications of Matrix-Assisted UV Laser Desorption/Ionization Mass Spectrometry. Anal. Chim. Acta 1990, $241,175-185$.

6. Rosinke, B.; Strupat, K.; Hillenkamp, F.; Rosenbusch, J.; Dencher, N.; Krüger, U.; Galla, H. J. Matrix-Assisted Laser Desorption/Ionization Mass Spectrometry (MALDI-MS) of Membrane Proteins and NonCovalent Complexes. J. Mass Spectrom. 1995, 30, 1462-1468.

7. Cohen, L. R. H.; Strupat, K.; Hillenkamp, F. Analysis of Quaternary Protein Assembles by Matrix-Assisted Laser Desorption/Ionization Mass Spectrometry. J. Am. Soc. Mass Spectrom. 1997, 8, 1046-1052.
8. Tang, X.; Callahan, J. H.; Zhou, P.; Vertes, A. Non-Covalent ProteinOligonucleotides Interactions Monitored by Matrix-Assisted Laser Desorption/Ionization Mass Spectrometry. Anal. Chem. 1995, 67, 45424548 .

9. Thiede, B.; von Janta-Lipinski, M. Non-covalent RNA-Peptide Complexes Detected by Matrix-Assisted Laser Desorption/Ionization Mass Spectrometry. Rapid Commun. Mass Spectrom. 1998, 13, 1889-1894.

10. Lin, S.; Long, S.; Ramirez, S. M.; Cotter, R. J.; Woods, A. S. Characterization of the "Helix Clamp" Motif of HIV-1 Reverse Transcriptase Using MALDI-TOF MS and Surface Plasmon Resonance. Anal. Chem. 2000, 72, 2635-2640.

11. Juhasz, P.; Bienmann, K. Mass Spectrometric Molecular Weight Determination of Highly Acidic Compounds of Biological Significance via Their Complexes with Basic Polypeptides. Proc. Natl. Acad. Sci. U.S.A. 1994, 91, 4333-4337.

12. Woods, A. S.; Buchsbaum, J. C. Worrall, T. A., Berg, J. M.; Cotter, R. J. Matrix-Assisted Laser Desorption/Ionization of Non-covalently Bound Compound. Anal. Chem. 1995, 67, 4462-4665.

13. Schlosser, G.; Pocsfalvi, G.; Malorni, A.; Puerta, A.; Frutos, M.; Vékey, K. Determination of Immune Complexes by Matrix-Assisted Laser Desorption/Ionization Mass Spectrometry. Rapid Commun. Mass Spectrom. 2003, 17, 2741-2747.

14. Glocker, M. O.; Bauer, S. H. J.; Kast, J.; Volz, J.; Przybylski, M Characterization of Specific Noncovalent Protein Complexes by UV Matrix-Assisted Laser Desorption/Ionization Mass Spectrometry. J. Mass Spectrom. 1996, 31, 1221-1227.

15. Zehl, M.; Allmaier, G. Ultraviolet Matrix-Assisted Laser Desorption/ Ionization Time-of-Flight Mass Spectrometry of Intact Hemoglobin Complex from Whole Human Blood. Rapid Commun. Mass Spectrom. 2004, 18, 1932-1938.

16. Parsons, J. F.; Calabrese, K.; Eisenstein, E.; Ladner, J. E. Structure and Mechanism of Pseudomonas aeruginosa PhzD, an Isochorismatase from the Phenazine Biosynthetic Pathway. Biochemistry 2003, 42, 5684-5693.

17. DeLange, R. J.; Huang, T. S. Egg White Avidin. III. Sequence of the 78-Residue Middle Cyanogen Bromide Peptide. Complete Amino Acid Sequence of the Protein Subunit. J. Biol. Chem. 1971, 246, 698-709.

18. Light-Wahl, K. J.; Schwartz, B. L.; Smith, R. D. Observation of Protein Quaternary Associations of Proteins by Electrospray Ionization Mass Spectrometry. J. Am. Chem. Soc. 1994, 116, 5271-5278.

19. Zehl, M.; Allmaier, G. Instrumental Parameters in the MALDI-TOF Mass Spectrometric Analysis of Quaternary Protein Structure. Anal. Chem. 2005, 77, 103-110.

20. Sundberg, E. I.; Sawicki, M. W.; Southwood, S.; Anderson, P. S.; Sette, A.; Mariuzza, R. A. Minor Structural Changes in a Mutated Human Melanoma Antigen Correspond to Dramatically Enhanced Stimulation of a CD4 ${ }^{+}$Tumor-infiltrating Lymphocyte Line. J. Mol. Biol. 2002, 319, $449-461$.

21. Kiselar, J. G.; Downard, K. M. Preservation and Detection of Specific Antibody-Peptide Complexes by Matrix-Assisted Laser Desorption/ Ionization Mass Spectrometry. J. Am. Soc. Mass Spectrom. 2000, 11, 746-750.

22. Borchers, C.; Tomer, K. B. Characterization of the Non-Covalent Complexes of Human Immunodeficiency Virus Glycoprotein 120 with Its Cellular Receptor by Matrix-Assisted Laser Desorption/Ionization. Biochemistry 1999, 38, 11734-11740.

23. Jespersen, S.; Niessen, W. M. A.; Tjaden, U. R.; van der Greef, J. Basic Matrices in the Analysis of Non-Covalent Complexes by MatrixAssisted Laser Desorption/Ionization Mass Spectrometry. J. Mass Spectrom. 1998, 33, 1088-1093.

24. Song, F. Patent pending.

25. Benjamin, D. R.; Robinson, C. V.; Hendrich, J. P.; Hartl, F. U.; Dobson, C. M. Mass Spectrometry of Ribosomes and Ribosomal Subunits. Proc. Natl. Acad. Sci. U.S.A. 1998, 95, 7391-7395. 